Asmaa Basher Al-Saffar BDS, MSc (Lec)

\section{Determination of the Real Distance of Mandibular Third Molar to Inferior Alveolar Canal}

\author{
Department of oral and Maxillofacial surgery \\ College of Dentistry, University of Mosul
}

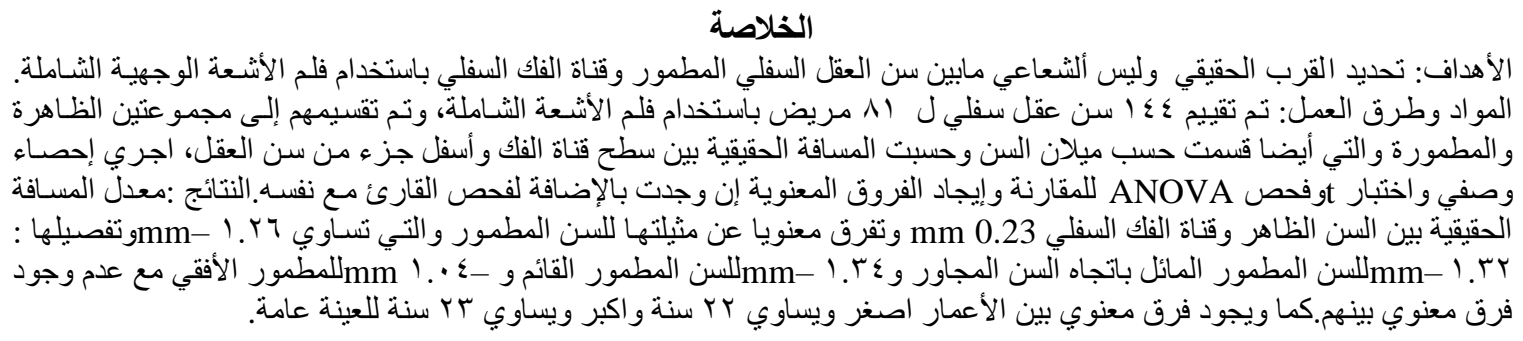

\begin{abstract}
Aims: To determine the real not radio graphical proximity of mandibular third molar tooth to the inferior dental canal (IDC) using panoramic radiograph. Materials and Methods: 144 mandibular third molars were evaluated by panoramic radiography. The teeth were grouped into erupted vs. un erupted further subdivided by tooth angulations. The real distance from the most inferior aspect of the mandibular third molar tooth to the superior border of the inferior alveolar canal (IAC) was calculated regarding to the reference object used. Descriptive statistics were performed as well as a t test was performed to compare erupted and unerupted teeth, and ANOVA was used to determine a significant difference where exists based upon tooth angulations. In addition, intra observer analysis was done to ensure the standardization of the radiologist interpretation. Results: The mean distance from erupted mandibular third molar teeth to the inferior alveolar canal was $0.23 \mathrm{~mm}$. This distance was significantly different from unerupted teeth $(\mathrm{P}=.000)$. The mean values for unerupted teeth were negative values which indicated that the apices of all teeth measured was below the superior border of the canal $-1.26 \mathrm{~mm}$ and as follows: Mesioangular-1.32 $\mathrm{mm}$, vertical $1.34 \mathrm{~mm}$ and $-1.04 \mathrm{~mm}$ for horizontal impactions. Statistically there was no significant positional difference between the impaction groups $(P=.835)$. In general there was a significant difference in third molar position between those age equal and less than 22 years old and those equal and over 23 years old. Conclusions: Unerupted mandibular third molar teeth (mostly vertical impaction) are closer to the inferior alveolar canal than erupted teeth and persons in general of an age equal or less than 23 years old have a closer lower third molar to mandibular canal than other ages and there is no significant relation between this age group and any of impaction types.
\end{abstract}

Key words: Third molar, inferior alveolar canal, proximity, panoramic radiograph.

Al -Saffar AB, Determination of the Real Distance of Mandibular Third Molar to Inferior Alveolar Canal. Al-Rafidain Dent J. 2016; 16(1):19- 29.

Received: 17/4/2011 Sent to Referees: 9/5/2011 Accepted for Publication: 8/10/2013

\section{INTRODUCTION}

The removal of impacted mandibular third molars teeth means teeth that fail to erupt into its functional position, (1) is one of the most common surgical procedures performed and can be complicated by inferior alveolar nerve (IAN) damage. (2) Anatomically, the mandibular nerve lies in 
the inferior dental canal which is enclosed within a tube of dense bone. As shown in Figure (1).

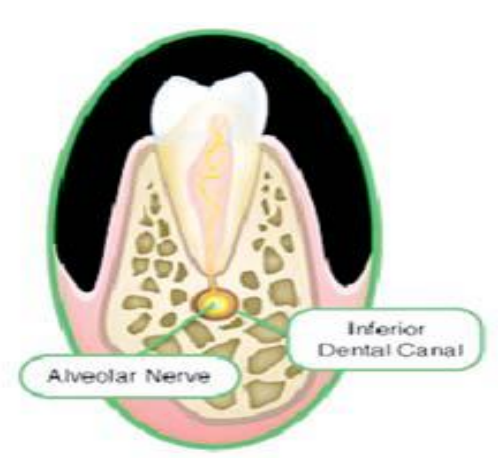

Figure(1): mandibular nerve in the IAC. ${ }^{(3)}$

The tube is seen on radiographs as two parallel radiopaque lines; one representing the roof of the canal and other the canal floor. ${ }^{(3)}$ Many authors have reported the incidence of post-operative dysaesthesia or impairment of sensory perception including paraesthesia and/or anesthesia of the IAN after extraction of third molars. (4-7) Temporary injuries in $0.4-5.5 \%$ and permanent nerve damage in $0.1-1.0 \%$ of cases of third molar extraction have been reported. ${ }^{(5)}$ Injury to IAN has been related to deeply impacted teeth and to roots in close approximation to the IDC; ${ }^{(3)}$ thus, a pre-operative radiographic assessment is required to identify approximation of IDC to third molar to minimize the risk of postoperative dysaesthesia . ${ }^{(8)}$ Rood and Shehab described 7 diagnostic signs to predict nerve injury when evaluating patients for possible extraction of third molars when the roots appear to be in close proximity to the mandibular canal (most importantly, darkening of the root, interruption of the white line of the IAC, and diversion of the IAC). (9) The radiographical relationship of the root apex of mandibular third molars to IDC was assessed and categorized according to the following criteria:

1- Adjacent : The superior border of the canal was either touching the roots apices or within $2 \mathrm{~mm}$ below them. 2- Superimposed : The canal was superimposed over part of the roots which appeared less radiopaque than the remaining radiological root's images.

3- Notching: Radiolucent band at the apex of the roots, a break in the continuity of the upper radio dense border, and narrowing at the expense of the top of the canal. 4- Grooving: Radiolucent band across the root above the apex, interruption of both superior and inferior borders of the canal and narrowing of the canal space. 5- Perforation: Radiolucent band crossing the root above the apex with loss of both superior and inferior borders of the canal at the area where they cross the roots and constriction of the canal maximal in the $\mathrm{m} \mathrm{i} \mathrm{d} \mathrm{l} \mathrm{e} \mathrm{o} \mathrm{f} \mathrm{th}$ e $\mathrm{r}$ o o t . 6- None: A relationship between the canal and the root apices could not be decisively assessed. ${ }^{(3)}$ More studies confirmed an association between these specific radiographic findings and IAN paresthesia. ${ }^{(6,10)}$ Nortje et al. ${ }^{(11)}$ who 
reviewed 3612 panoramic radiographs and found the position of the IDC was either touching or within $2 \mathrm{~mm}$ of the apices of molar teeth in $46.7 \%$ of the subjects; in $48.9 \%$ the IDC was touching or within $2 \mathrm{~mm}$ of the cortical plate of the lower border of the mandible and intermediately positioned between tooth apices and the lower border in $3.3 \%$ of $\mathrm{th} \mathrm{e} \quad \mathrm{s} \mathrm{u} \mathrm{b} \mathrm{j} \mathrm{e} \mathrm{c} \mathrm{t} \mathrm{s.}$ Various preoperative radiographic techniques to evaluate the relationship between the mandibular third molar and the IDC can be used, these include: intra-oral radiographs, OPGs, cross sectional tomography, scan graphs, and $\mathrm{CTs}{ }^{(3,12)}$ the panoramic radiography is the optimum method for radiological assessment for mandibular third molar teeth prior to their removal, ${ }^{(6)}$ as well as it offers both excellent anatomical assessments and excellent evaluations of jaw fractures, tooth development, with availability of modifications to handicapped patients as well as those with gag reflex sensitivity. ${ }^{(13)}$ However, a panoramic radiography is a two dimensional (2D) image, lacking information in the bucco-lingual direction and magnification. ${ }^{(14)}$

The purpose of this study is to determine the mean real distance of the most inferior portion of the mandibular third molar to the superior border of the IDC for both erupted and impacted teeth.

\section{MATERIALS AND METHODS}

Panoramic radiographs of 144 lower third molars of 81 patients (45 males and 36 females) who attended the maxillofacial unit in College of Dentistry, University of Mosul were taken for examination of lower third molar. Some patients had uni /or bi lateral impacted or erupted third molars, the age ranged between16-48 years. Of the 144 teeth included in the study, 49 were erupted third molars in 16-39 years old patients and 95 were impacted in the age range 16-48 years divided into 47 mesioangular, 26 vertical and 22 horizontal angulations. Both sides of jaw were examined on the radiograph to calculate the proximity of third molar to IDC. These radiographs met the following inclusion criteria: Erupted or unerupted mandibular third molars with a mandibular second molar present; erupted teeth with an opposing tooth in the maxillary arch. The reason for this was to prevent wide variation that resulted from drifting of an erupted third molar with root displacement. The following were exclusion criteria: Displacement of the tooth due to pathology such as cysts or tumors, extracted adjacent mandibular second molar, not well processed radiographic film, impacted third molar of distal or inverted types because they were few in number (only 2 inverted and 4 distal) out of hundred impacted molars and because the wanted number of these two rare types were unavailable and could not obtained to perform a true statistical methods, or accepted comparison with other impacted groups, they were excluded from the study. Conventional Panoramic imaging system which more available and used in medical centers than digital one, panoramic radiographs performed with Ortho pantamography (STARTO X2000), Italy with exposure parameters of $70 \mathrm{kvp}, 10$ $\mathrm{mA}, 15$ Sec using intensifying screen film type Agfa and Cevaert extra oral film. The

film was processed manually in adark room according to manufactures instruction.

Each OPG was examined under ideal conditions including the use of subdued light and magnifying lens. The radiographs were viewed on a radiographic view box $12 \mathrm{mAx}$ 
by a single examiner. The impacted mandibular third molars were classified according to the long axis - occlusal plane angle; determination of the angle between the occlusal plane or a line parallel to it and longitudinal axis of impacted third molars within the corresponding Winter subclasses as follows:

1. Third molars with an angle between $0^{\circ}$ to $30^{\circ}$ are considered horizontal.
2. Third molars with an angle between $31^{\circ}$ to $60^{\circ}$ are considered mesioangular.

3. Third molars with an angle between $61^{\circ}$ to $90^{\circ}$ are considered vertical. ${ }^{(15)}$

Manual caliper (ruler) was used to measure on panoramic radiograph the radiographic length of the reference object ( radiopaque wire) of known length fixed on patient's cheek using adhesive strip against the ramus area of mandible , as shown in Figure (2)

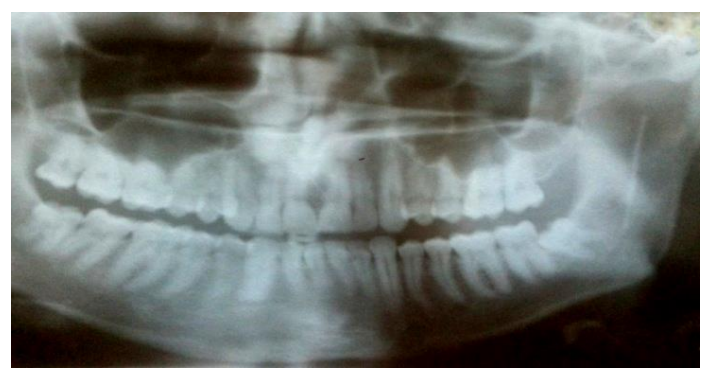

Figure(2): Conventional panoramic radiograph showing radiopaque reference object near mesially angulated mandibular third molar(left side)

To measure the radiographic distance from the superior cortical plate of IDC to the most inferior part of the third molar which in the majority of cases root apex, or in very few numbers a portion of the crown (in ahorizantal or mesioangular impaction), or per coronal follicle of the tooth (in teeth with incompletely formed roots). In instances where the tooth landmark was in a position that was inferior to the superior border of the IDC, a negative value was recorded, the linear magnification of panoramic image $(\mathrm{m})$ which is the ratio of image size to object size was calculated using radigraphic and real length of reference object, then by substitution, the study calculated the anatomical real distance from IDC to third molar using the known mathematic equation or relation $:\{\mathrm{m}=$ Radiographic length of wire / Real length of wire $=$ Radiographic distance between IDC and tooth / Real distance between IDC and tooth\}. ${ }^{(16)}$ In order to determine the accuracy of the radiographic measurements by the examiner, on the OPG, 10 randomly chosen radiographs in which measurements were made from the lower portion of mandibular third molar to the IAC and values were recorded. One week following the initial evaluation, these radiographs were again measured and recorded. These values were used to determine intra observer variability. The teeth were grouped into 4 categories, depending on the angulations of the tooth and whether it was erupted or impacted (erupted, mesioangular, vertical and horizontal). Descriptive statistics, ANOVA as well as a standardized $t$ test were performed to compare the erupted to 
impacted molars, and a second $t$ test was done to compare between types of impactions using Duncan's Multiple Range Test. We attempt to assess the radiographs with regard to age and gender.

RESULTS

The mean distance from the most inferior part of mandibular third molar to IAC was, on average, positive value (root apices above IAC) in erupted teeth and a negative value (root apices below IAC) to impacted teeth, mostly the vertical group, as shown in Figure (3) and Tables $(1,2)$.

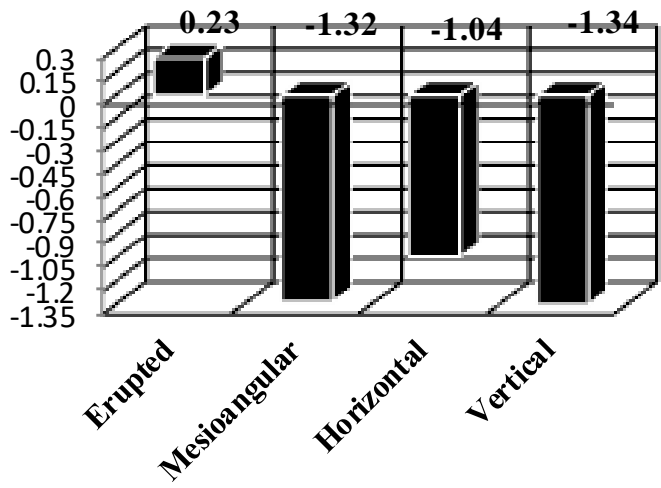

Figure(2): The mean distance from mandibular third molar to IAC.

Table (1): Distance in(mm.) from mandibular canal to mandibular third molar.

\begin{tabular}{llllll}
\hline Eruption & Number & Minimum & Maximum & Mean & Std.Deviation \\
Erupted & 49 & -4.54 & 5.60 & 0.23 & 2.23 \\
Impacted & 95 & -5.45 & 3.47 & -1.26 & 2.00 \\
\hline
\end{tabular}

Table (2): Distance in(mm.) from mandibular canal to Impacted third molar.

\begin{tabular}{llllll}
\hline Imp. type & Number & Minimum & Maximum & Mean & Std.Deviation \\
Mesioangular & 47 & -5.45 & 3.47 & -1.32 & 1.928 \\
Horizontal & 22 & -5.45 & 2.67 & -1.040 & 2.171 \\
Vertical & 26 & -5.35 & 3.12 & -1.346 & 2.063 \\
\hline
\end{tabular}


The $t$ test showed a very high significant difference between erupted and impacted mandibular third molars in general $(P=$ $.000)$. ANOVA also was performed to compare the results of impacted groups to determine whether there was a significant difference in distances between the individual classifications of impaction and the results disclosed with no significant difference $(p=0.83)$, owing to the lack of difference noted by the mean values and standard deviations. Duncan's Multiple Range Test performed showed no significant difference between impaction groups. A $t$ test showed no significant differences in the mean distance between males and females of erupted ( $p=0.83$ ), or impacted teeth groups $(p=0.55)$ as shown in Table (3).

Table (3): Distance in(mm.) from mandibular canal to mandibular third molar regarding to gender and eruption.

\begin{tabular}{lllllll}
\hline Eruption & Sex & Number & Minimum & Maximum & Mean & Std. Deviation \\
& & & & & & \\
\hline Erupted & Male & 29 & -4.54 & 5.60 & 0.18 & 2.45 \\
& Female & 20 & -2.27 & 5.33 & 0.32 & 1.94 \\
Impacted & Male & 54 & -5.45 & 3.47 & -1.16 & 2.04 \\
& Female & 41 & -5.35 & 3.12 & -1.40 & 1.96 \\
\hline
\end{tabular}

Regarding to side differences, the results showed no significant differences between the right and left side of erupted $(p=0.26)$ and impacted (0.97) as shown in Table (4)

Table (4) Distance in (mm.) from mandibular canal to mandibular third molar regarding to side and eruption

\begin{tabular}{ccccccc}
\hline Eruption & Side & Number & Minimum & Maximum & Mean & Std.Deviation \\
Erupted & Right & 27 & -4.54 & 5.60 & 0.56 & 2.39 \\
& Left & 22 & -3.63 & 4.20 & -0.15 & 2.01 \\
Impacted & Right & 46 & -5.45 & 3.47 & -1.26 & 2.17 \\
& Left & 49 & -5.45 & 3.12 & -1.27 & 1.85 \\
\hline
\end{tabular}


The results differed regarding to age, as shown in Table (5), in general the $t$ test showed a significant difference in the mean distance between patients of ages over and equal to 23 years and those equal and less than 22 years of age $(p=0.025)$. Although there were differences but it was not significant between these two age groups with in erupted or impacted $(p=0.916),(p=0.88)$ respectively as shown in Table (6).

Table (5) Distance in(mm.) from mandibular canal to mandibular third molar regarding to age in years(y.) in the whole sample.

\begin{tabular}{rccccc}
\hline Age & Number & Minimum & Maximum & Mean & Std.Deviation \\
$<=\mathbf{2 2} \mathbf{y}$. & 99 & -5.45 & 5.60 & -1.03 & 2.13 \\
$>=\mathbf{2 3} \mathbf{y}$. & 45 & -4.54 & 5.33 & -0.41 & 2.24 \\
\hline
\end{tabular}

Table (6) Distance in(mm.) from mandibular canal to mandibular third molar regarding to Age in years(y.) and eruption.

\begin{tabular}{lcccccc}
\hline Eruption & Age/y & Number & Minimum & Maximum & Mean & Std.Deviation \\
Erupted & $<=\mathbf{2 2 y}$. & 27 & -4.09 & 5.60 & 0.27 & 2.23 \\
& $>=\mathbf{2 3 y}$. & 25 & -4.54 & 5.33 & 0.20 & 2.28 \\
\multirow{2}{*}{ Impacted } & $<=\mathbf{2 2 y}$. & 75 & -5.45 & 3.12 & -1.44 & 1.93 \\
& $>=\mathbf{2 3 y}$. & 20 & -4.46 & 3.47 & -0.58 & 2.16 \\
\hline
\end{tabular}

Regarding Intra observer examination ,the paired samples $t$ test showed no significant difference found between first and second radiographic interpretation $(p=0.168)$.

\section{DISCUSSION}

One of the complications that may occur following the extraction of mandibular third molars is injury to the IAN, ${ }^{(3)}$ but it is uncommon. ${ }^{(17)}$

The purpose of a careful radiological evaluation is to complement the clinical examination by providing additional information about the third molar, the related teeth and anatomical features, and the surrounding bone. This is necessary in order to make a sound decision about the proposed surgical procedure, the most appropriate location for this to take place, and to highlight aspects of management which may require specific mention to the patient. $^{(18)}$

Panoramic and periapical radiographs are usually used during surgery of impacted third molar teeth to study the condition of the teeth with regard to the angle and direction of the third molar in relation to the occlusal plane or to the second molar direction. Although panoramic radiography is used in the location of the third molars, extensive diseases and developmental anomalies, it cannot present the details like periapical radiographs. ${ }^{(19)}$

When more than one third molar requires to be assessed, the radiographic examination of choice is a panoramic radiograph as the radiation dose of a panoramic radiograph is lower than from 
four periapical views and the diagnostic yield higher. Doses from panoramic radiography can be further limited by using field size limitation to prevent exposing areas not required in the field of view. Periapical or oblique lateral radiographs may be taken as an alternative. ${ }^{(18)}$ The results of the Atieh $\mathrm{A}^{(20)}$ study, suggest a reasonable diagnostic accuracy for panoramic radiography in the preoperative evaluation of the relationship between third molars and the canal.

The results of this study as expected showed that the erupted third molars were usually farther from the IAC than impacted third molars. Vertical impactions were located most inferiorly with respect to the superior border of the IAC, followed by the mesioangular and farthest was horizontal impaction group, based on the distances measured in this study. Statistically no significant difference was found between impaction groups regarding the distance from the IAC and each of these impaction groups on panoramic radiographs. With this information, impacted mandibular third molars are more likely to be located in a position that may place the IAN at risk during third molar odontectomy mostly vertical type of impaction as it has average proximity of $-1.346 \mathrm{~mm}$ below the IAC. This agrees with Hazza'a et al. ${ }^{(3)}$ results in which vertical impacted molars were mainly found to be in true relationship with the IDC followed by the mesial, and horizontal. This suggests that surgeons should be careful when interpreting the radiographs and operating on vertically impacted teeth. Never the less, the obtained negative values not necessary represent a danger in all the times because it represented and resulted from many types of the relationships between third molar and IDC like superimposition or grooving relations as they previously mentioned, not from magnifications or radiographic deformity because the current study excluded the magnification and calculated the real distance using the radiographic distance on panoramic film.

The conducted study agreed with apart of miloro et al. ${ }^{(21)}$ conclusions that there is a significant positional difference between erupted and impacted teeth $(\mathrm{p}=.002)$ and disagreed with their findings that mesioangular impactions were significantly different than other impaction groups $(\mathrm{p}=.012)$ and it is most closely positioned to IAC (-0.97) followed by vertical (-0.61) and horizontal (-0.24). It is believed that this difference aroused because of the racial population difference and the radiographic values found were not real compared to this study.

For the whole sample of patients age who represent the Mosul collages' students age (about 22 years and less) or dental collags' patients ( mainly above and equal 23 years old), despite they provide the study parameters, either impacted or erupted third molars, there was statically a significant difference in the distances means between those age $\geq 23$ years and those of $\leq 22$ years old, the last group have shown more proximallity to IAC than other one.

Although the most accurate method of prediction with precision of the position of the IAN pre-operatively is the use of computerized tomography providing more complete information than did the conventional radiographs. ${ }^{(6)}$ Nevertheless, the conventional equipment of computed tomography was not originally developed for dental use, ${ }^{(22)}$ the relatively high radiation exposure, high cost, the need for space, the long exposition time are drawbacks of CTs. ${ }^{(23)}$ Therefore, it can be concluded that OPG remains the most 
common radiograph used for the assessment of impacted mandibular third molars and appears to have the best cost-information ratio. ${ }^{(3,24)}$

\section{CONCLUSIONS}

Vertical mandibular third molar impactions are most closely positioned to the inferior alveolar canal than other types of impactions or erupted, and this may represent an independent risk factor for post operative paraesthesia. Persons in general of an age equal to or over 23 years of age have a closer lower third molar to the mandibular canal than other ages and there is no significant relation between this age group and any of impaction type.

\section{REFERENCES}

1. Pedlar J, Frame JW .Oral and Maxillofacial Surgery. $2^{\text {nd }} \quad$ ed. Edinburgh London Newyork Oxford Philadelphia Stlouis Sydney Toronto. 2007 ;P : 46-47.

2. Carmichael FA, McGowan DA. Incidence of nerve damage following third molar removal a West of Scotland Oral Surgery Research Group study. $\mathrm{Br}$ J Oral Maxillo fac Surg. 1992;30:78.

3. Hazza'a AM, Albashaireh ZS, Bataineh $A B$. The relationship of inferior dental canal to the roots of impacted mandibular third molars in Jordanian population. Cont dent pract. 2006;2 (7): 071-078.

4. Öhman A, Kivijärvi K, Blombäck U ,Flygare L. Pre-operative radiographic evaluation of lower third molars with computed tomography. Dent maxillo fac Rad. 2006 ;35: 30-35.

5. Valmaseda-Castellon E, Berini-Aytes L, Gay-Escoda C. Inferior alveolar nerve damage after lower third molar surgical extraction: A prospective study of 1117 surgical extractions. Oral Surg. 2001; 92: 377-383.

6. Smith AC, Barry SE, Chiong AY, Hadzakis D, Kha SL, Mok SC, Sable DL. Inferior alveolar nerve damage following removal of mandibular third molar teeth. A prospective study using panoramic radiography. Aust Dent J. 1997; 42(3): 149-152.

7. Loescher A R, Smith K G, Robinson P P. Nerve damage and third molar removal. Dent Update. 2003;30:375382.

8. Koong B, Pharoah MJ, Bulsara M, Tennant M. Methods of determining the relationship of mandibular canal and third molars: A survey of Australian oral and maxillofacial surgeons. Aust Dent J. 2006;51(1): 64-68.

9. Rood JP, Shehab BA. The radiological prediction of inferior alveolar nerve injury during third molar surgery. $\mathrm{Br} J$ Oral Maxillofac Surg.1990;28:20-25.

10. Rud J. Third molar surgery: Relationship of root to mandibular canal and injuries to the inferior dental nerve. Tandlaegebladets. 1983; 87:619-631. 
11. Nortje CJ, Farman AG, Joubert JJ.The radiographic appearance of the inferior dental canal. An additional variation. $\mathrm{Br}$ J Oral Surg.1977;15:171-172.

12. Kaeppler G. Conventional crosssectional tomographic evaluation of mandibular third molars. Quintessence Int.2000;(31): 49-56.

13. Yiu B K, Liu S C, Ng P C, Siu G H. Digital dental panoramic radiography: Evaluation of image quality in four imaging systems. Hong Kong Dent J. 2005;2:19-23.

14. Faleh W, Zahrani A. Observer Agreement On Radiographic Assessment of Mental Foramen Appearance In Panoramic Radiographs. J, Oral Maxillofac. radiolo. 2005; 25(2): 225- 228.

15. Almendros MN, Berini AL, Gay EC. Influence of lower third molar position on the incidence of post operative complication .Oral Surg.2006; 102: 725 3.

16. Giambattista A, Richardson B, , Richardson R, Physics. Mc Graw Hill. 2008;p861-862.

17. Chkoura A, El Wady W, Taleb B.Third molar and mandibular canal: selection of radiographic techniques]. Rev Stomatol Chir Maxillofac. 2011;112(6):360-4.

18. Management of Unerupted and Impacted Third Molar Teeth. 2013; duncan.service@nhs.net.
19. Ardakani FE, Booshehri MZ, Behniafar B. Evaluation of the Distortion Rate of Panoramic and Periapical Radiographs in Erupted Third Molar Inclination. Iran J Radiol. 2011; 8(1):15-21. ( IVSL ).

20. Atieh MA. Diagnostic accuracy of panoramic radiography in determining relationship between inferior alveolar nerve and mandibular third molar. $J$ Oral Maxillofac Surg. 2010;68(1):7482.

21. Miloro M, DaBell J. Radiographic proximity of the mandiular third molar to inferior alveolar canal. Oral Surg. 2005; 100: 545-9.

22. Junior O F, Avila L D, Sampieri M B S, Rabeiro E D, Chen W, Fan S. Impacted lower third molar fused with a supernumerary tooth-Diagnoses and treatment planning using Cone-beam computed tomography. Int J Oral Sci. 2009;1(4):224-228.

23. Monaco G, Montevecchi M, Bonetti GA,Rosaria M, Gatto A, Checchi L. Reliability of panoramic radiography in evaluation relationship between the mandibular canal and impacted third molars. J Am Dent Assoc. 2004; 135:312-318.

24. Kositbowornchai S, Densiri-aksorn W, Piumthanaroj P. Ability of two radiographic methods to identify the 
closeness between the mandibular third molar root and the inferior alveolar
25. canal: a pilot study. Dentomaxillo fac Radiol. 2010;39(2):79-84. 